\title{
Human trophoblast-derived exosomes attenuate doxorubicin-induced cardiac injury by regulating miR-200b and downstream Zeb1
}

Jie $\mathrm{Ni}^{1+}$, Yihai Liu ${ }^{1 \dagger}$, Lina Kang ${ }^{2}$, Lian Wang ${ }^{2}$, Zhonglin Han ${ }^{2}$, Kun Wang ${ }^{2^{*}}$, Biao Xu ${ }^{1,2^{*}}$ and Rong $\mathrm{Gu}^{2^{*}}$

\begin{abstract}
Human trophoblast stem cells (TSCs) have been confirmed to play a cardioprotective role in heart failure. However, whether trophoblast stem cell-derived exosomes (TSC-Exos) can protect cardiomyocytes from doxorubicin (Dox)induced injury remains unclear. In the present study, TSC-Exos were isolated from the supernatants of human trophoblasts using the ultracentrifugation method and characterized by transmission electron microscopy and western blotting. In vitro, primary cardiomyocytes were subjected to Dox and treated with TSC-EXos, miR-200b mimic or miR200b inhibitor. Cellular apoptosis was observed by flow cytometry and immunoblotting. In vivo, mice were intraperitoneally injected into Dox to establish a heart failure model. Then, different groups of mice were administered either PBS, adeno-associated virus (AAV)-vector, AAV-miR-200b-inhibitor or TSC-Exos via tail vein injection. Then, the cardiac function, cardiac fibrosis and cardiomyocyte apoptosis in each group were evaluated, and the downstream molecular mechanism was explored. TSC-Exos and miR-200b inhibitor both decreased primary cardiomyocyte apoptosis. Similarly, mice receiving TSC-ExOs and AAV-miR-200b inhibitor exhibited improved cardiac function, accompanied by reduced apoptosis and inflammation. The bioinformatic prediction and luciferase reporter results confirmed that Zeb1 was a downstream target of miR-200b and had an antiapoptotic effect. TSC-Exos attenuated doxorubicininduced cardiac injury by playing antiapoptotic and anti-inflammatory roles. The underlying mechanism could be an increase in Zeb1 expression by the inhibition of miR-200b expression. In summary, this study sheds new light on the application of TSC-Exos as a potential therapeutic tool for heart failure.
\end{abstract}

Keywords: Trophoblasts, Exosomes, miR-200b, Heart failure

\section{Introduction}

Cancer mortality has decreased during recent decades due to the widespread use of anthracyclines [1]. However, the number of patients suffering from the longterm effects of cardiotoxicity has increased [2, 3]. Several

\footnotetext{
*Correspondence: kingwang726@163.com; xubia062@nju.edu.cn; gurong. nju@163.com

†Jie Ni and Yihai Liu contributed equally to this work

1 Department of Cardiology, Nanjing Drum Tower Hospital, Clinical

College of Nanjing Medical University, Nanjing 210008, Jiangsu, People's Republic of China

${ }^{2}$ Department of Cardiology, Affiliated Drum Tower Hospital, Medical School of Nanjing University, Nanjing 210008, Jiangsu, People's Republic of China
}

agents used in cancer treatment, such as doxorubicin (Dox), can adversely affect cardiac function and lead to congestive heart failure, which is a leading cause of disability in long-term survivors of cancer $[4,5]$ and a growing problem in the field of cardio-oncology.

Dox is an anthracycline cytostatic agent that has been in clinical use for almost a half century [6-8]. However, cumulative doses of Dox were found to be harmful to the myocardium [9], leading to left ventricular dysfunction and the development of heart failure, namely, Doxinduced cardiotoxicity. The underlying mechanisms include mitochondrial iron accumulation and reactive oxygen species (ROS) burst [10-13], eventually leading to cell apoptosis or cell necrosis [14-18]. However, the exact 
mechanisms have not been fully established, and optimal cardioprotective treatments remain undefined [8].

Various types of stem cells have been proposed for use in cardiac cell therapy $[19,20]$, such as skeletal myoblasts, mesenchymal stem cells (MSCs), hematopoietic stem cells (HSCs), and embryonic stem cells (ESCs). Trophoblast stem cells (TSCs) are a population of stem cells that originate from a single layer of blastocysts when the earliest cellular differentiation occurs [21]. Our previous results [22] illustrated that TSCs could improve cardiac function and attenuate myocardial adverse remodeling, which could be attributed to a lower level of miRNA200b-3p. However, stem cell therapy is limited by an inconsistent supply, infusion toxicity, low survival and immune rejection. In addition, researchers confirmed that the cardioprotective effect of the administered stem cells was not derived from direct transdifferentiation into cardiomyocytes. Rather, this effect was mediated by the paracrine effects of exosomes secreted by stem cells [23, 24]. Therefore, in our study, we explored the effect of trophoblast cell-derived exosomes on Dox-induced cardiac injury and the underlying mechanism.

\section{Materials and methods}

\section{Animal experiments}

C57BL/6 mice (male, 8 weeks) were purchased from the Model Animal Research Center of Nanjing University. The animals were fed a standard laboratory diet with free access to food and water and housed in a temperature $\left(22 \pm 1^{\circ}\right)$ - and humidity (65-70\%)-controlled room with a 12-h light-dark cycle. After the study, all the animals were anesthetized by isoflurane inhalation $(1.5-2 \%)$ and then euthanized by cervical dislocation. All the procedures with animals were approved by the Institutional Ethics Committee of Nanjing Drum Tower Hospital (Approval No. 20011141) and performed in accordance with the Care and Use of Laboratory Animals published by the National Institutes of Health (Eighth Edition).

Doxorubicin (Dox; Cat: D1515; Sigma; USA) was intraperitoneally administered to mice at a dose of $5 \mathrm{mg} / \mathrm{kg}$ once a week for 4 weeks (Dox groups). The Dox+Exo groups received an additional intramyocardial injection of $25 \mu \mathrm{l}$ of PBS containing $50 \mu \mathrm{g}$ TSC-Exos. The Dox +AAV groups received a tail vein injection of $25 \mu \mathrm{l}$ $\left(1 * 10^{\wedge} 11\right.$ v.g.) of adeno-associated virus serotype 9 (AAV9; GeneChem; Shanghai), which carried miR200b inhibitor (a small RNA to silence the expression of miR-200b), while the Dox+Vector groups received an equivalent dose of empty virus. All the groups were anesthetized by isoflurane inhalation (1.5-2\%), ventilated with a rodent ventilator and subjected to thoracotomy for intramyocardial injection at 3 different points. Each group included 5 mice.

\section{Exosome isolation and identification}

TSCs (HTR8-Svneo; Novobio; Shanghai) were cultured in DMEM containing 10\% FBS and 1\% penicillin/streptomycin (Gibco; USA) solution in $5 \% \mathrm{CO}_{2}$ at $37{ }^{\circ} \mathrm{C}$. When the cells reached 70 to $80 \%$ confluency, they were cultured with fresh DMEM containing 5\% exosome-depleted fetal bovine serum (Cat: 161004-001; System Biosciences Inc; USA) and incubated for $48 \mathrm{~h}$. Exosomes were isolated using differential centrifugation based on previously described methods with slight modifications $[25,26]$. Briefly, the cells were harvested by centrifugation at $300 \times g$ for $10 \mathrm{~min}$; the supernatant was then cleared of apoptotic bodies by centrifugation at $2000 \times g$ for $20 \mathrm{~min}$; MVs were preferentially pelleted at $10,000 \times g$ for $30 \mathrm{~min}$; and exosomes were then purified from the supernatant by ultracentrifugation at $100,000 \times g$ for $60 \mathrm{~min}$. After isolation, $10 \mu \mathrm{l}$ exosomes were diluted in $1 \mathrm{ml}$ filtered PBS and stored at $-80^{\circ} \mathrm{C}$.

The morphology of the exosomes was observed using a transmission electron microscope (JEM1011; Japan), and the size distribution was measured using nanoparticle tracking analysis (NTA). For electron microscopy, we used $0.5 \%$ uranyl acetate to stain the exosomes and calculated their diameter from 5 to 15 images. For NTA, the samples were loaded into the sample chamber of an NS500 unit (Nanosight, Amesbury, UK), and five 1 -min videos of each sample were recorded. Data analysis was performed with NTA 2.3 software (Nanosight), and the size and concentration of particles were calculated. Exosome markers (CD9, CD63 and TSG101) were detected by immunoblotting. To facilitate tracking in vitro, exosomes were labeled with the PKH67 Red Fluorescent Cell Linker Kit (Cat: PKH67GL; Sigma; USA).

\section{Cardiomyocyte culture and treatment}

Primary cardiomyocytes were isolated from neonatal mice (post 1-2 days; Science of Chinese Academy of Medical Sciences; Beijing). In brief, hearts were enzymatically digested in HEPES-buffered saline solution containing $1.2 \mathrm{mg} / \mathrm{ml}$ pancreatin and $0.14 \mathrm{mg} / \mathrm{ml}$ collagenase at $37{ }^{\circ} \mathrm{C}$. After centrifugation, the cells were collected and resuspended in DMEM. The dissociated cells were preplated at $37^{\circ} \mathrm{C}$ for $1 \mathrm{~h}$ and then diluted to $1^{*} 10^{6}$ cells $/ \mathrm{ml}$ and plated in $10 \mathrm{mg} / \mathrm{ml}$ laminin-coated culture dishes according to the specific experimental requirements. The Dox group received doxorubicin $(1 \mu \mathrm{M})$ for $24 \mathrm{~h}$. The Dox+Exo group received an additional $20 \mu \mathrm{g}$ of exosomes. In addition, the cells were treated with miR200b mimic (50 nM) (Dox+Mimic) and miR-200b inhibitor (50 nM) (Dox+Inhibitor), which were synthesized to overexpress miR-200b and inhibit miR-200b, respectively. 


\section{Echocardiography}

Cardiac function was assessed by transthoracic echocardiography (VEVO2100; visual sonics). The mice were anesthetized with isoflurane (1.5\% in air) and monitored for respiratory frequency and temperature. End diastole was measured at the time of the apparent maximal LV diastolic dimension, and end systole was measured at the time of the most anterior systolic excursion of the posterior wall. The mice were assessed at 1 week before and 1 , 2, 3 and 4 weeks after TSC-Exos injection. Left ventricular internal dimensions at end-diastole (LVIDd) and endsystole (LVIDs) were digitally measured on the M-mode tracings from 3 cardiac cycles. The left ventricular ejection fraction (EF) and left ventricular fractional shortening (FS) were calculated accordingly.

\section{RNA extraction and real-time RT-PCR}

Total mRNA was extracted using a commercial kit (Cat: 9108Q; TaKaRa; Japan) according to the manufacturer's instructions. Quantitative analyses were carried out on a real-time system (Applied Biosystems; USA) using SYBR Green PCR Master Mix (Cat: Q331-01; Vazyme, China) for mRNA or miRNA Universal SYBR qPCR Master Mix for miRNA (Cat: MQ101-01; Vazyme, China). The miRNA primers were obtained from Genescript (Nanjing). The relative expression level for each mRNA was calculated using the $2^{-\Delta \Delta C t}$ method. The primers were as follows: ANP F: GCTTCCAGGCCATATTGGAG; R: GGGGGCATGACCTCATCTT; $\beta M H C$ F: ACTGTC AACACTAAGAGGGTCA; R: TTGGATGATTTGATC TTCCAGGG; and Collagen I F: GCTCCTCTTAGG GGCCACT; R: CCACGTCTCACCATTGGGG.

\section{Western blotting}

Heart tissues, exosomes and cells were lysed using RIPA buffer (Beyotime; Jiangsu). The protein concentration was measured by the BCA method (Thermo; USA). Equal amounts of protein were loaded on SDS-PAGE gels, separated and then transferred onto PVDF membranes (Millipore; USA). Then, the membranes were incubated with primary rabbit anti-mouse antibodies against CD9 (Cat: ab92726), CD63 (Cat: ab213090), TSG101 (Cat: ab125011), cleaved-caspase3 (Cat: ab2302), Bcl-2 (Cat: ab32124), Zeb1 (Cat: ab81972), p65 (Cat: ab16502) and GAPDH (Cat: ab181602) (Abcam; USA) at a dilution of 1:1000. After overnight incubation at $4{ }^{\circ} \mathrm{C}$, the membranes were subsequently incubated with HRP-conjugated rabbit anti-mouse IgG (1:10000) for $1 \mathrm{~h}$ at room temperature. The immunobands were visualized using an enhanced chemiluminescence (ECL) detection kit (Cat: P0018S; Beyotime; China).

\section{Annexin V/PI staining for cell apoptosis}

Primary cardiomyocytes were digested and collected at a concentration of $1^{*} 10^{\wedge} 6 \mathrm{cells} / \mathrm{ml}$. Then, the cells were resuspended in $200 \mu \mathrm{l}$ binding buffer, and $5 \mu \mathrm{l}$ AnnexinFITC and PI were added (Cat: KGA108-2; Keygen; China). After incubating for $30 \mathrm{~min}$ in the dark, the cells were analyzed by flow cytometry (BD; USA).

\section{Enzyme-linked immunosorbent assay (ELISA)}

Murine blood was collected from the retro-orbital sinus and centrifuged at $3000 \mathrm{rpm}$ for $5 \mathrm{~min}$ at $4{ }^{\circ} \mathrm{C}$ to acquire serum. The serum IL-1 $\beta$ (Cat: MLB00C) and IL-6 (Cat: M6000B) levels were measured using commercial enzyme-linked immunosorbent assay kits according to the manufacturer's instructions (R\&D; UK).

\section{Luciferase reporter measurement}

The luciferase reporter clones with the $3^{\prime}$-untranslated region (UTR) of Zeb1 were purchased from GeneCopoeia (Rockville; MD). The clones included predicted miR-200b target sites acquired from an online database (www.mirdb.org) and a mutated sequence of the $3^{\prime}$ UTR of Zeb1. The plasmids were transfected into HEK293 cells (200 ng per well) using Lipo 2000 (Life Technologies; USA) with $10 \mathrm{nM}$ miR-200b mimic. After $24 \mathrm{~h}$, the cell medium was collected for the luciferase assay.

\section{Histology assay}

Heart tissues were harvested after 4 weeks. Then, the heart tissues were fixed in $4 \%$ paraformaldehyde $(\mathrm{pH}$ 7.4). After fixation and paraffin embedding, the cardiac tissues were cut into 5 - $\mu \mathrm{m}$-thick sections. The sections were stained with hematoxylin and eosin (Servicebio; China) to analyze the global heart morphology and inflammatory cell infiltration. The sections were stained with Masson's trichrome (Servicebio; China) to evaluate cardiac fibrosis. The sections were scanned at 20-fold magnification on a high-resolution microscope (Leica; Japan).

\section{Statistical analysis}

Data are presented as the mean \pm standard derivation. All statistical analyses were performed with SPSS (23.0). Differences were analyzed with one-way analysis of variance (ANOVA) for multiple groups and Student's t-test for only two groups. $\mathrm{p}<0.05$ was considered statistically significant.

\section{Results}

Characterization of TSC-derived exosomes

Utilizing ultracentrifugation, we obtained exosomes from TSCs, as shown by TEM (Fig. 1a). The 
a

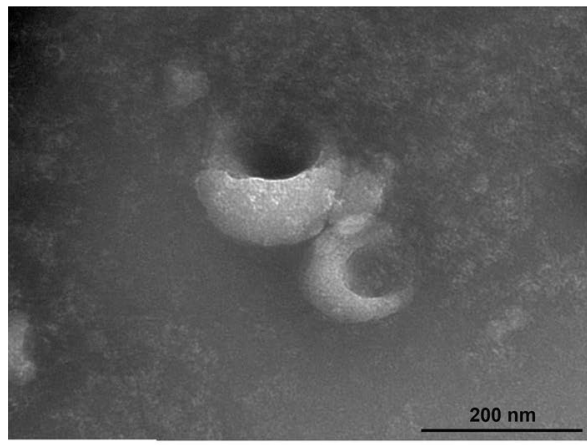

b

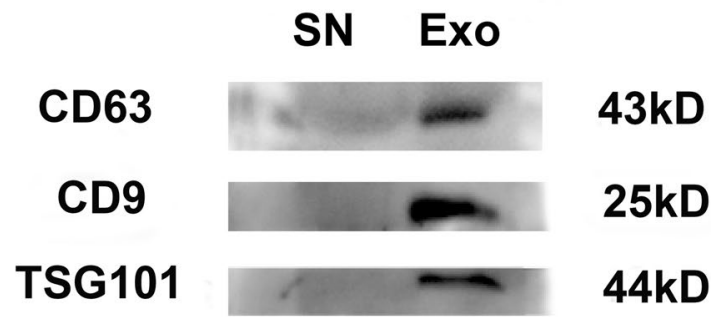

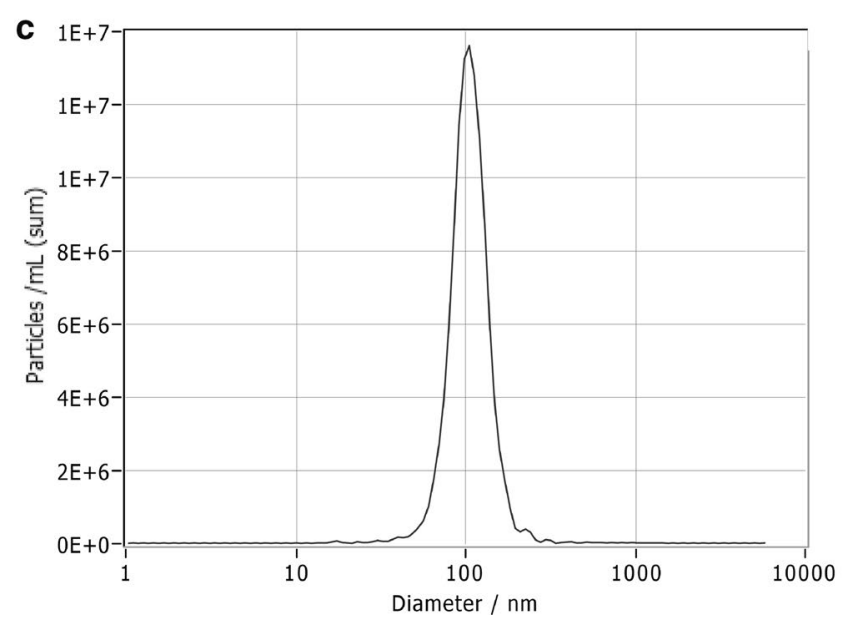

Fig. 1 Characterization of TSC-derived exosomes. a The ultrastructure of exosomes was analyzed by transmission electron microscopy. b Western blot analysis showed the expression of protein markers of exosomes, such as CD63, CD9 and TSG101. c Representative DLS number distribution measurement of the isolated exosome population demonstrated an average diameter of $101 \mathrm{~nm}$

immunoblotting results (Fig. 1b) confirmed the expression of exosomal markers (CD63, CD9, and TSG101). In addition, DLS illustrated that the exosomes had an average diameter of $101 \mathrm{~nm}$ (Fig. 1c).

\section{TSC-derived exosomes protected cardiomyocytes from Dox-induced apoptosis}

As shown in Fig. 2a, labeled exosomes were taken up by primary cardiomyocytes. Previous studies showed that TSCs could decrease the expression of miR-200b in cardiac tissues [22]. Therefore, we treated primary cardiomyocytes with TSC-Exos as well as miR$200 \mathrm{~b}$ mimic and inhibitor. The flow cytometry results (Fig. 2b) showed that exosomes and inhibitors both inhibited cell apoptosis. In addition, exosome- and inhibitor-treated cardiomyocytes had lower C-caspase 3 expression and higher Bcl-2 expression than the cardiomyocytes in other groups (Fig. 3).

\section{TSC-Exos improved cardiac function in Dox-induced heart} failure

Dox-treated mice were treated with or without exosomes to determine the effect of exosomes in vivo. After 4 weeks, the echocardiographic results (Additional file 1: Table S1) showed that exosome-treated mice had a higher EF and FS (Fig. 4a, b), suggesting a favorable role of the exosomes in cardiac remodeling. Additionally, heart failure-associated markers (ANP, $\beta$-MHC, and Collagen I) showed decreased expression in the exosome group (Fig. 4c).

\section{TSC-Exos decreased miR-200b expression and played a protective role in vivo}

miR-200b was downregulated in both TSC-Exo-treated cardiac tissues and primary cardiomyocytes (Fig. 5a, b), suggesting that miR-200b is involved in the protective role of TSC-Exos. To further confirm the role of miR-200b, miR-200b was inhibited by transfecting 


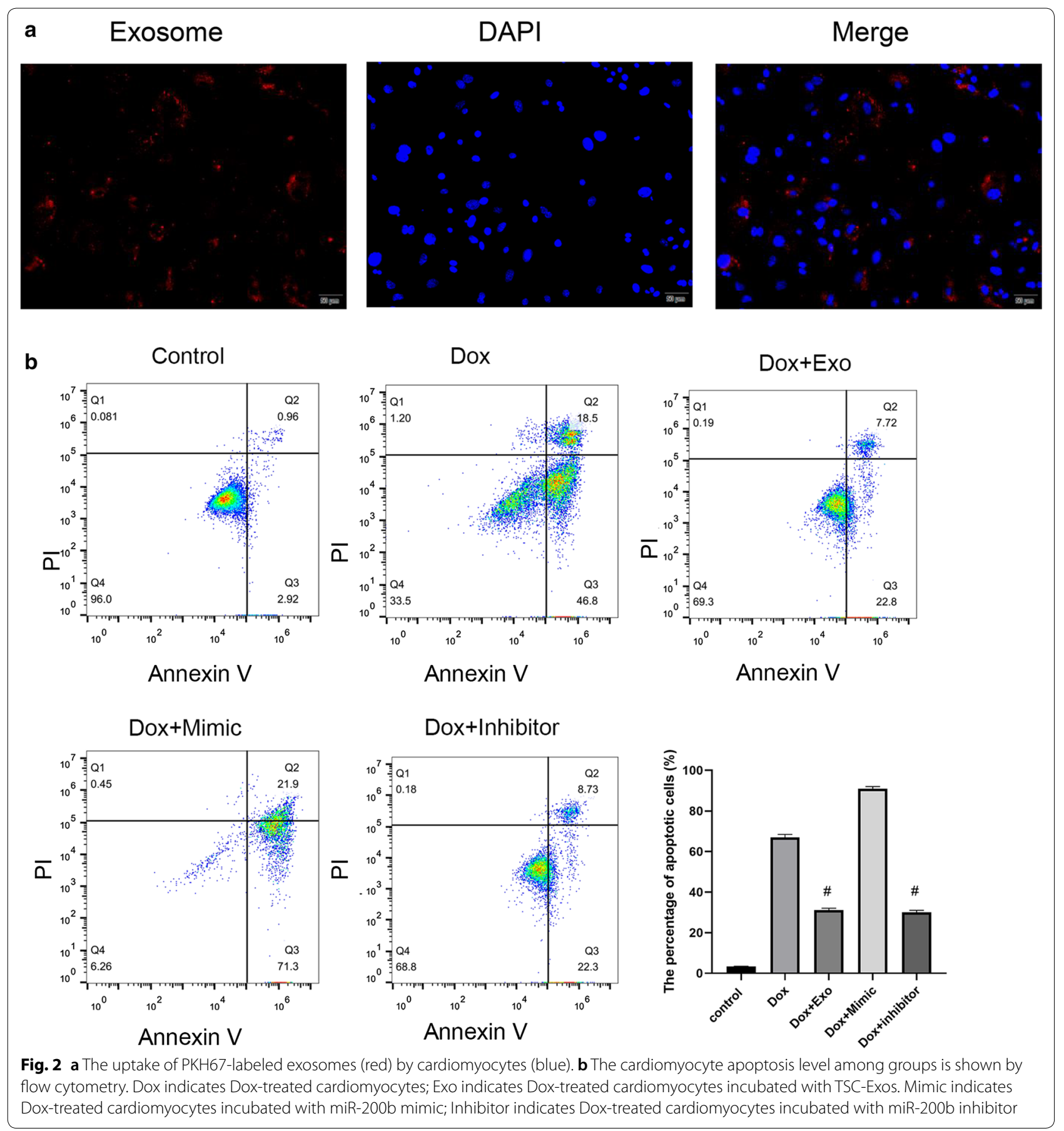

the miR-200b inhibitor AAV. The exosome group and inhibitor group exhibited decreased levels of serum IL-1 $\beta$ and IL-6 (Fig. 6a) and cardiac p65 (Fig. 6b), suggesting decreased systemic inflammation and local inflammation. Moreover, exosomes and miR-200b inhibitor both attenuated the heart size (Fig. 7a), the heart weight to body weight ratio (Fig. 7b), and cardiac fibrosis (Fig. 7c), suggesting a favorable role of these treatments in cardiac remodeling.

\section{Zeb1 was a target of miR-200b involved in the protective role of TSC exosomes}

Previous studies revealed that Zeb1 was a downstream target of miR-200b and exerted an antiapoptotic effect. 


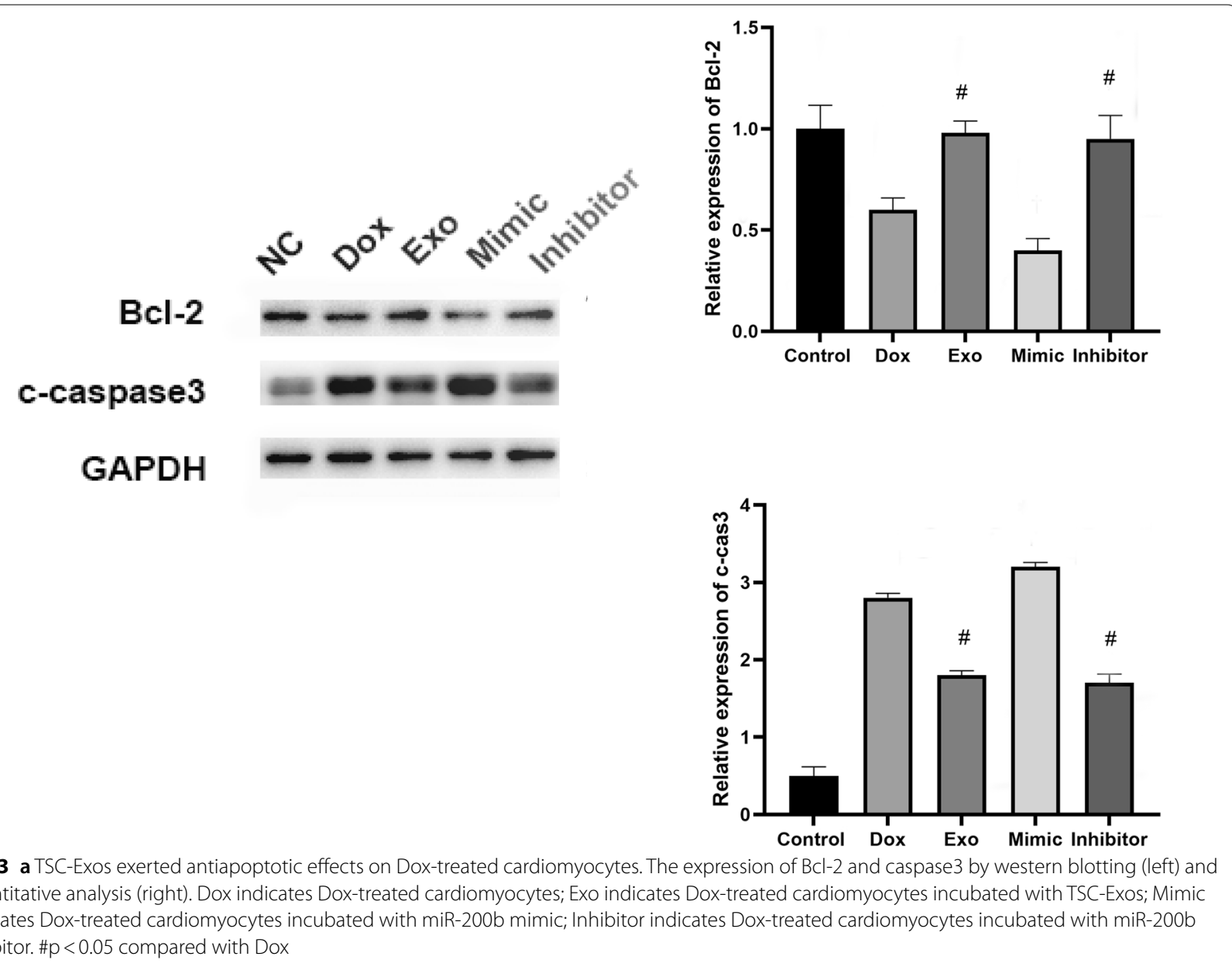

Therefore, we examined Zeb1 expression in the abovementioned groups. In addition to decreased apoptosis, the exosome and overexpression groups both exhibited increased Zeb1 expression (Fig. 8a). The luciferase reporter assay also confirmed the direct binding of miR-200b to Zeb1 (Fig. 8b).

\section{Discussion}

Our studies revealed, for the first time, that TSC-Exos can protect murine hearts from Dox-induced injury by regulating miR-200b expression. Specifically, TSC-Exos exerted an antiapoptotic and anti-inflammatory effect by decreasing the expression of Zeb1 and some inflammatory cytokines, which provided an alternative mechanism of reversing chemotherapy-induced heart failure.

MicroRNAs (miRNAs), as a class of small noncoding RNAs [27], have emerged as posttranscriptional regulators that function by binding to the $3^{\prime}$-UTR of target genes [28]. miRNAs are involved in cell proliferation, differentiation, metastasis, apoptosis, and immune responses [29]. Doxorubicin could cause cardiac injury by inducing the expression of some miRNAs. For example, a study confirmed a strong association of miR34a-5p and miR-451a with Dox-induced cardiotoxicity [30]. miR-21 suppression or miR-130a prevented cardiac dysfunction induced by doxorubicin [31, 32]. Altered miRNAs could be a potential biomarker or early toxicity signature, which was reviewed in some publications [33, 34]. In our study, the expression of miR-200b was upregulated in Dox-treated cardiomyocytes and was negatively correlated with the expression of $\mathrm{Bcl} 2$, indicating that miR-200b was a proapoptotic mediator. Our results showed that TSC-Exos decreased miR-200b expression in cardiomyocytes and inhibited apoptosis in cardiomyocytes by upregulating the anti-apoptotic Bcl-2 protein. miR-200 members were reported to promote cell apoptosis in cancer [26, 35], which was consistent with our result that miR-200 mimic aggravated cardiac injury suffering from Dox injection. Korpal et al. had previously reported that miR-200b could target Zeb1 [36]. A luciferase reporter was constructed to confirm the direct binding of miR-200b and Zeb1. However, it 

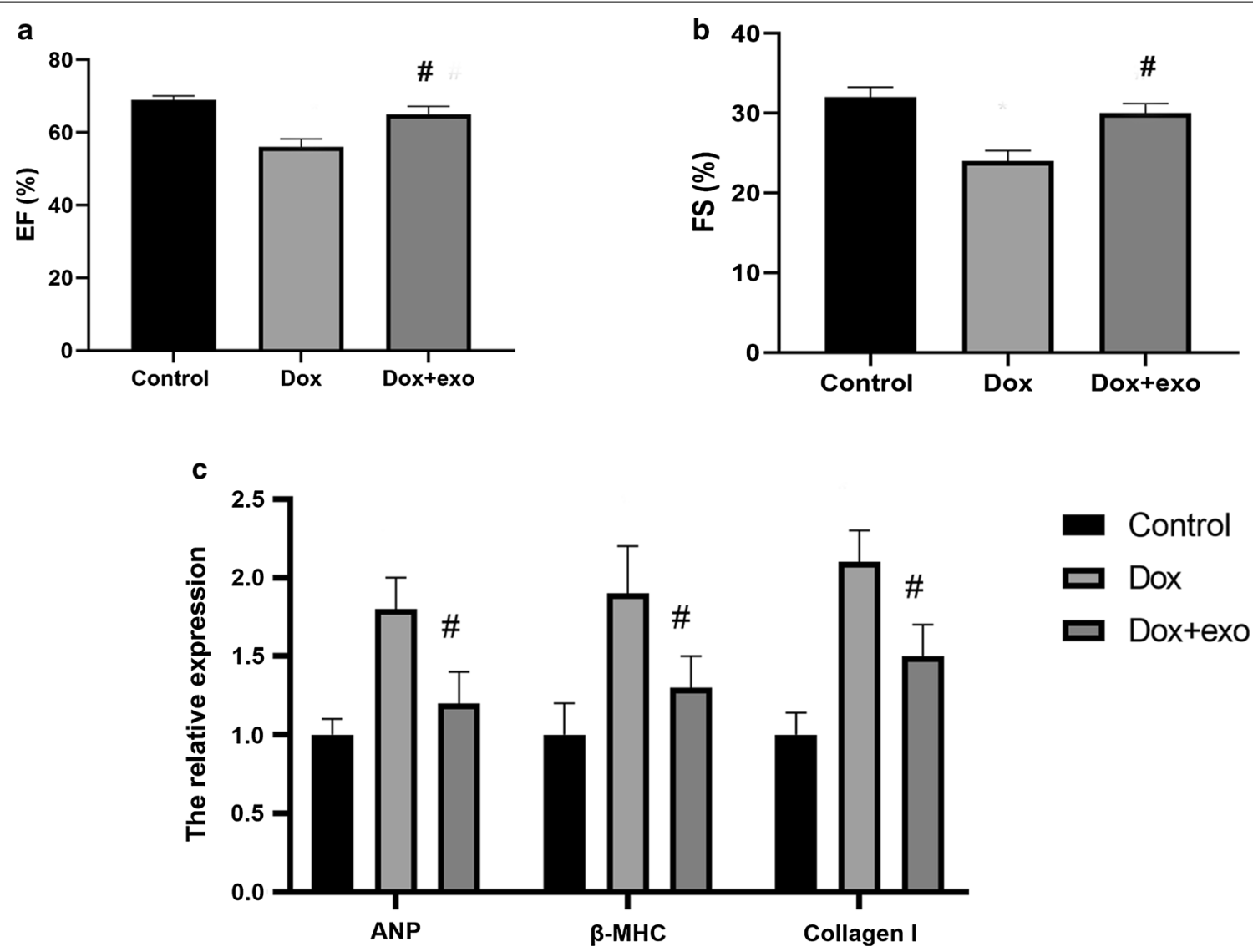

Fig. 4 Echocardiographic data of different groups after 4 weeks. a Left ventricular ejection fraction (EF); b left ventricular fraction shortening (FS); $\mathbf{c}$ mRNA expression of heart failure markers, such as ANP, MHC and Collagen I. Dox indicates Dox-treated mice injected with PBS; Dox+Exo indicates Dox-treated mice injected with TSC-Exos. \#p <0.05 compared with Dox

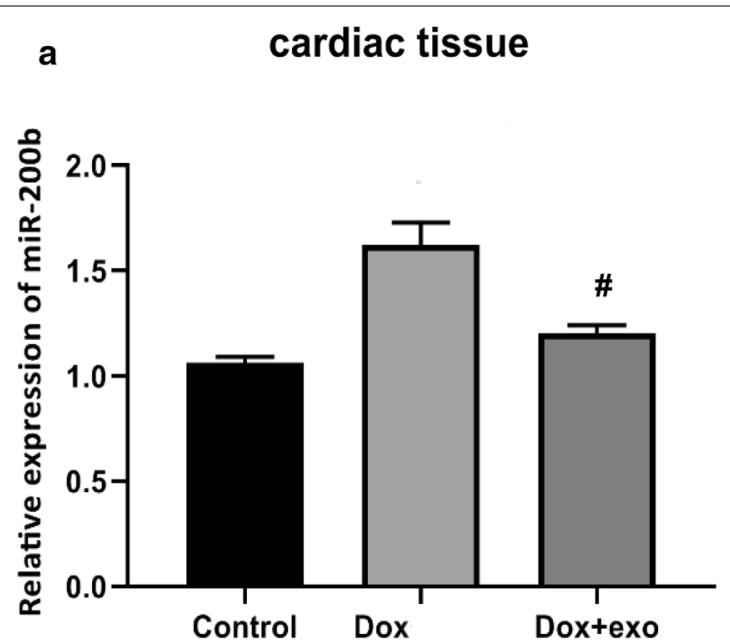

b

cardiomyocyte

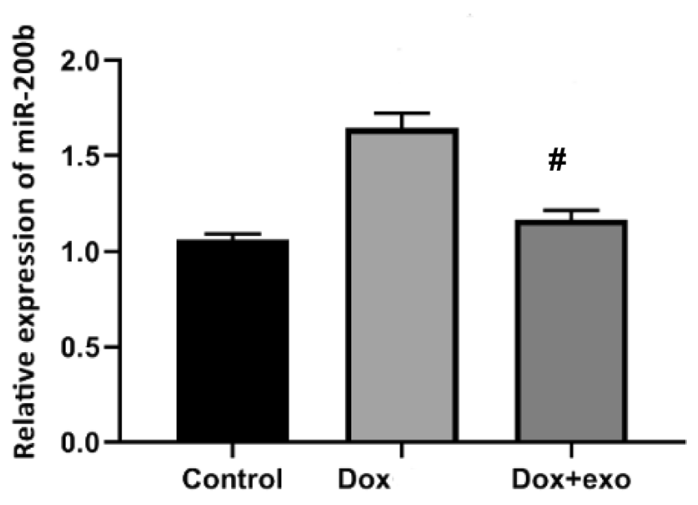

Fig. 5 a The expression of miR-200b in heart tissues of Dox-treated mice; $\mathbf{b}$ the expression of miR-200b in Dox-treated cardiomyocytes. Dox indicates Dox-treated mice injected with PBS; Dox+Exo indicates Dox-treated mice injected with TSC-Exos. \#p < 0.05 compared with Dox 
a

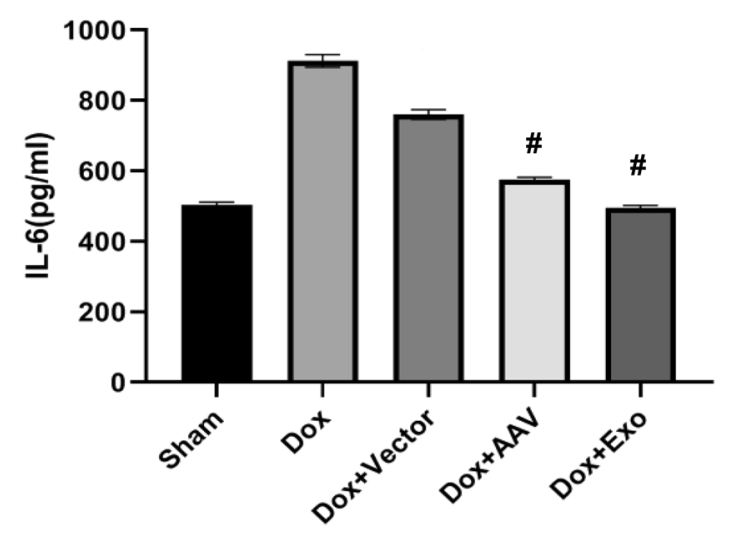

b

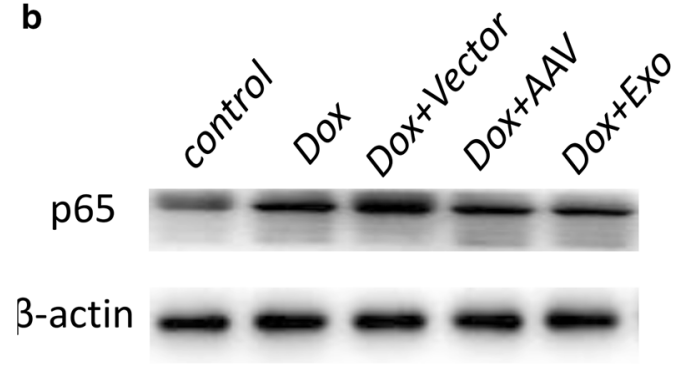

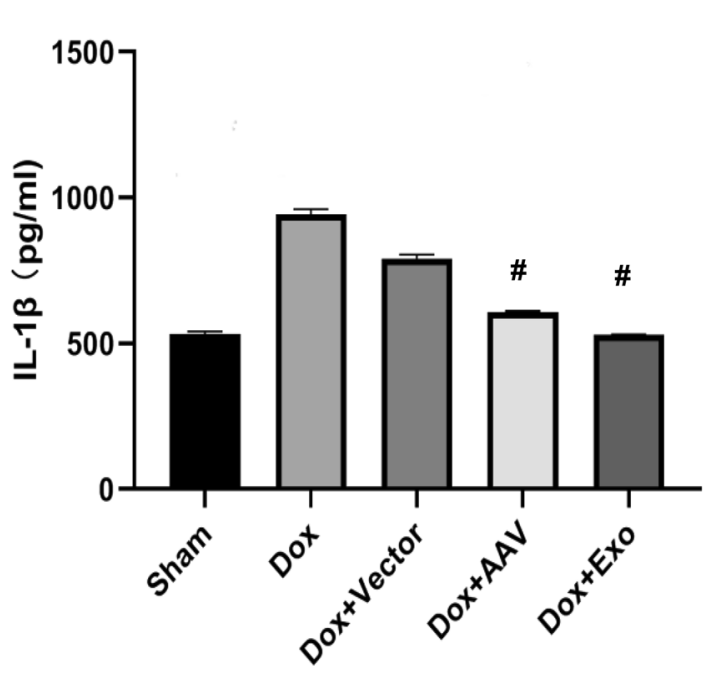

10
0
0
40
0
$\frac{0}{0}$
$-\frac{0}{n}$
0
0
0
$\frac{0}{x}$
0
0
0
$\frac{1}{5}$

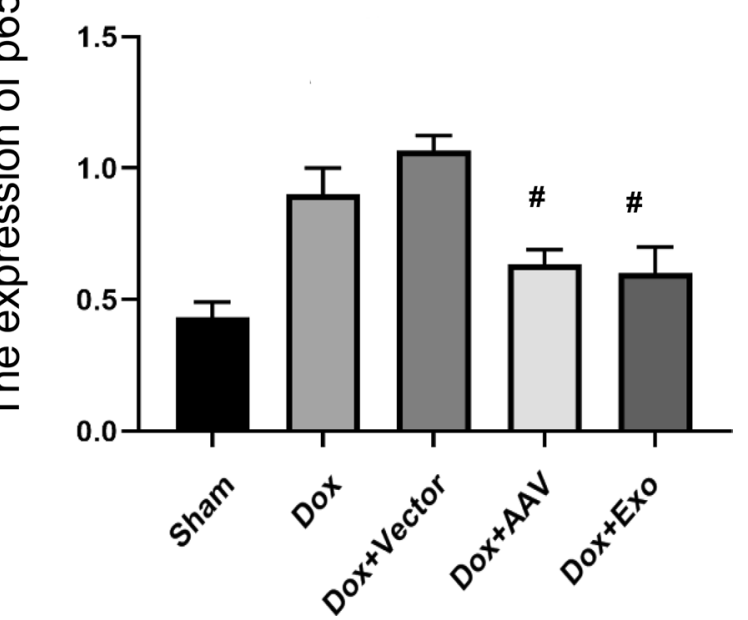

Fig. 6 TSC-Exos exert anti-inflammatory effects in Dox-induced cardiac injury. a ELISA analysis of the concentration of inflammatory factors, including IL-1 $\beta$ and IL-6; $\mathbf{b}$ the expression of $\mathrm{p} 65$ by western blotting. Dox+Vector indicates Dox-treated mice injected with AAV9 vector; Dox+AAV indicates Dox-treated mice injected with AAV9-miR-200b inhibitor; Dox+Exo indicates Dox-treated mice injected with TSC-Exos. \#p $<0.05$ compared with the Dox+Vector group

remains unclear how TSC-Exos regulate the expression of miR-200b. Some studies reported that TSC-Exos could play a role by delivering noncoding RNAs, proteins and cytokines to recipient cells [37-39]. We hypothesized that IncRNAs enriched in TSC-Exos could transfer to hearts and bind to and silence miR-200b via a ceRNAdependent mechanism, which needs to be confirmed by future experiments.

Recent findings illustrated that Dox activated the NF- $\mathrm{KB}$ pathway to cause inflammatory effects on the myocardium and vasculature [40]. Conversely, blocking the NF- $\mathrm{kB}$ pathway in cardiac microvascular endothelial cells could weaken inflammatory damage [41]. Our data showed that IL-1b, IL-6 and p65 were increased in the Dox and miR200b mimic groups but decreased in the exosome and inhibitor groups. In addition, we monitored echocardiography in animal models and found that cardiac function was significantly improved in animal models treated with exosomes. These results suggested that exosomes could inhibit the inflammatory response, thereby protecting cardiomyocytes and improving cardiac function subjected to Dox injury. 

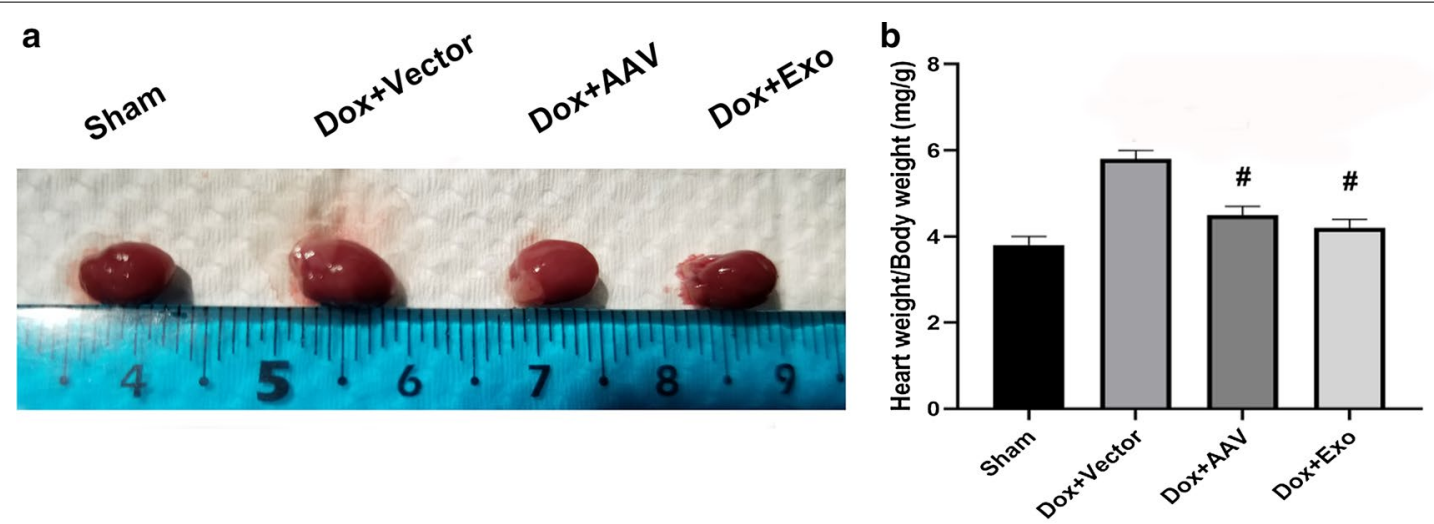

C

Sham

Dox+Vector
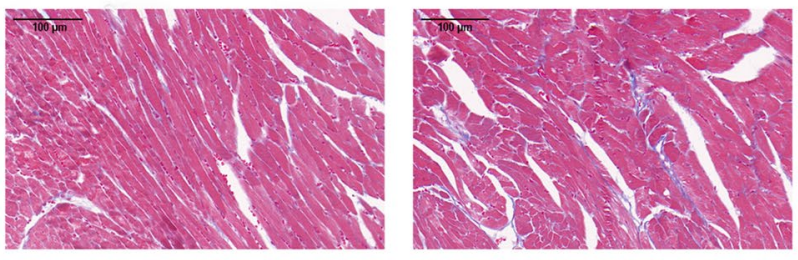

Dox+AAV

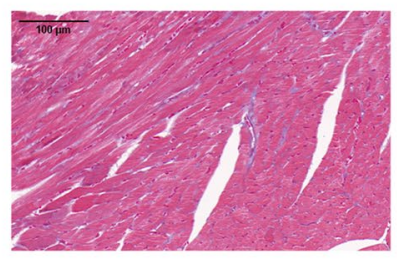

Dox+Exo

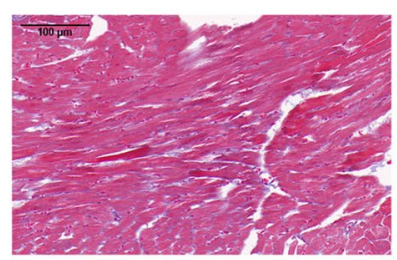

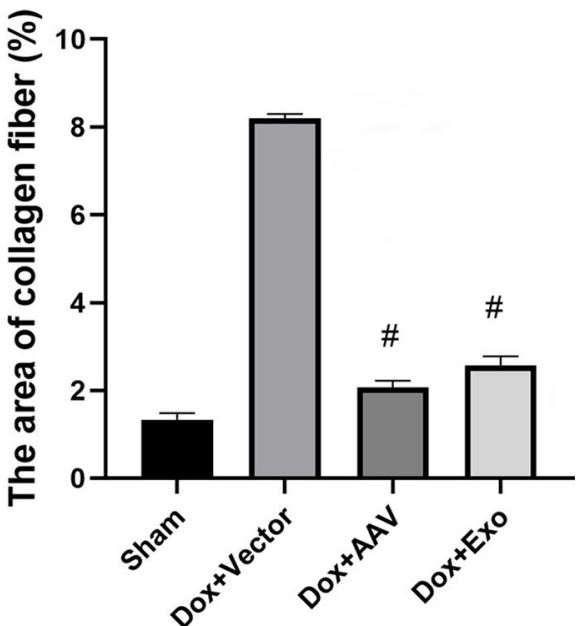

Fig. 7 a The morphology of the hearts of different groups; $\mathbf{b}$ the heart weight to body weight ratio of different groups; $\mathbf{c}$ the fibrotic areas of different groups by Masson staining. Right is $\times 20$ magnification of the corresponding area labeled with a black rectangle in the left whole LV ring. Dox+Vector indicates Dox-treated mice injected with AAV9 vector; Dox+AAV indicates Dox-treated mice injected with AAV9-miR-200b inhibitor; Dox+Exo indicates Dox-treated mice injected with TSC-Exos. \#p $<0.05$ compared with the Dox+Vector group

\section{Conclusion}

In summary, our study elucidated the antiapoptotic role of TSC-Exos in injured cardiomyocytes, which was achieved by inhibiting miR-200b while increasing Zeb1 expression. This study provides a new strategy to treat heart failure in the future. 
a

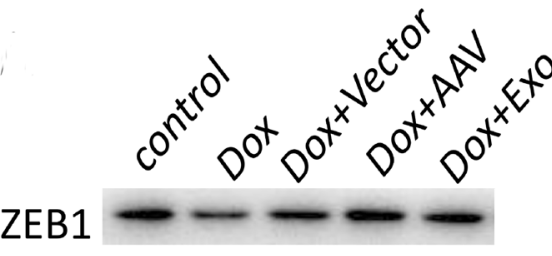

Caspase 3

$\mathrm{BCL} 2$

\section{$\beta$-actin}

b

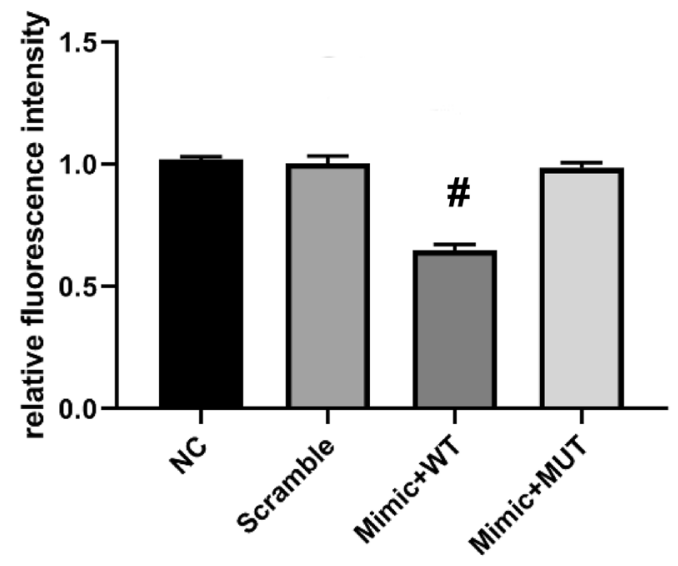

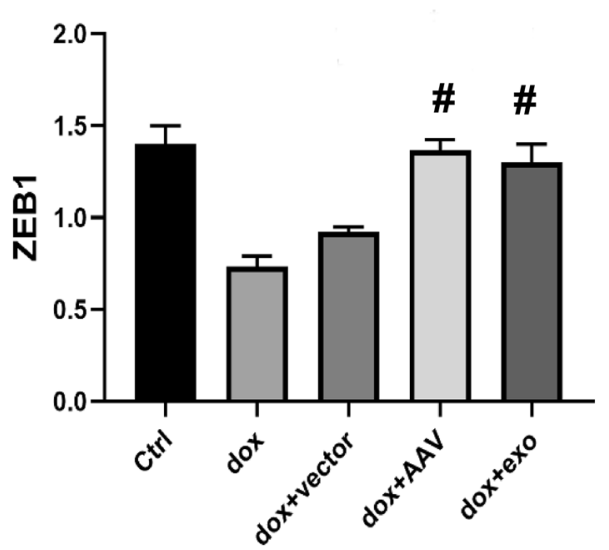
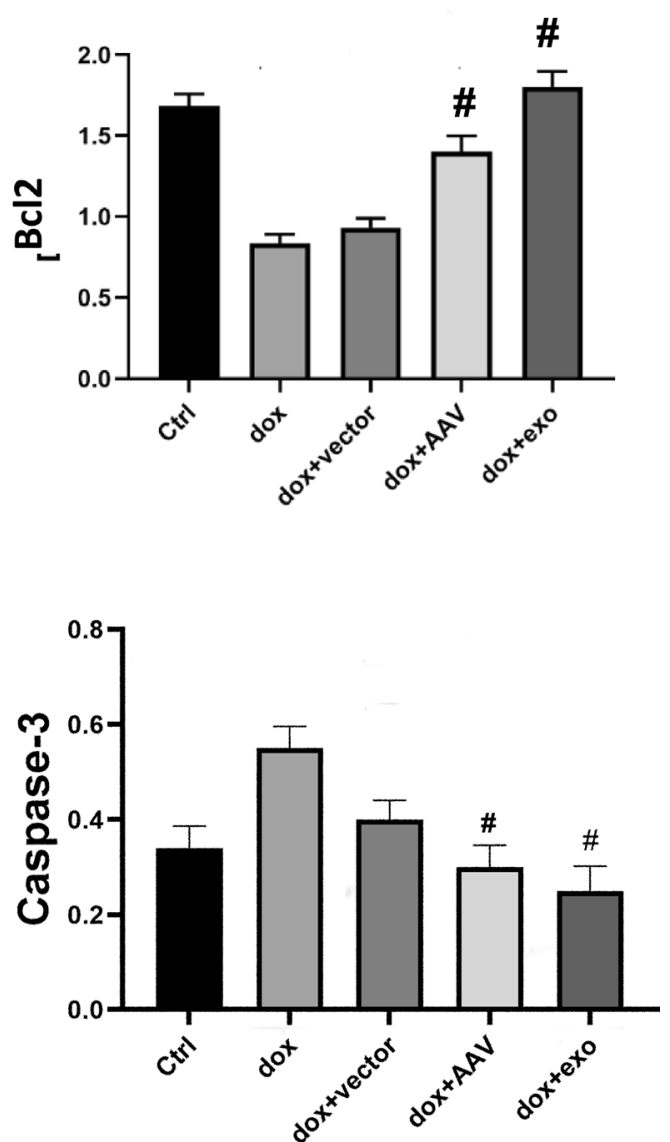

Fig. 8 TSC-Exos exert anti-apoptotic effects on tissues with Dox-induced cardiac injury. a The expression of Zeb1, Caspase3 and Bcl-2 was measured by western blotting, and $\beta$-actin was used as the reference control; the quantitative results are shown on the right. Dox indicates Dox-treated mice; Dox+Vector indicates Dox-treated mice injected with AAV9 vector; Dox+AAV indicates Dox-treated mice injected with AAV9-miR-200b inhibitor; Dox+Exo indicates Dox-treated mice injected with TSC-Exos. b The luciferase results showing miR-200b binding to Zeb1. Scramble indicates cells transfected with the scramble sequence; Mimic+WT indicates cells transfected with the miR-200b-3p and wild type 3'UTR of Zeb1; Mimic+MUT indicates cells transfected with the miR-200b-3p and mutated 3'UTR of Zeb1. \#p <0.05 compared with Dox or Scramble 


\section{Supplementary information}

Supplementary information accompanies this paper at https://doi. org/10.1186/s12951-020-00733-z.

Additional file 1: Table S1. The echocardiographic parameters of the control group, Dox group, Dox+Exo group, Dox+Vector group and Dox+AAV group ( $n=5$ each group).

\section{Abbreviations}

TSC: Trophoblast stem cells; TSC-Exos: Trophoblast stem cell-derived exosomes; Dox: Doxorubicin; AAV: Adeno-associated virus; ROS: Reactive oxygen species; MSCs: Mesenchymal stem cells; HSCs: Hematopoietic stem cells; ESCs: Embryonic stem cells; LVIDd: Left ventricular internal dimensions at end-diastole; LVIDs: Left ventricular internal dimensions at end-systole; EF: Ejection fraction; FS: Fractional shortening; ELISA: Enzyme-linked immunosorbent assay; UTR: 3'-Untranslated region; ANOVA: One-way analysis of variance.

\section{Acknowledgements \\ Not applicable.}

\section{Authors' contributions}

JN and YL drafted the article; LK, LW and ZH performed experiments; KW, RG and BX designed the study and approved the manuscript. All authors read and approved the final manuscript.

\section{Funding}

This research was supported by grants from the National Natural Science Foundation of China (Grant Nos. 81870291, 81600267 and 81870204).

\section{Availability of data and materials}

All the data can be obtained upon reasonable request.

\section{Ethics approval and consent to participate}

Not applicable.

\section{Consent for publication}

Not applicable.

\section{Competing interests}

The authors declare that they do not have anything to disclose regarding conflicts of interest with respect to this manuscript.

Received: 30 July 2020 Accepted: 13 November 2020

Published online: 20 November 2020

\section{References}

1. van Dalen EC, Raphael MF, Caron HN, Kremer LC. Treatment including anthracyclines versus treatment not including anthracyclines for childhood cancer. Cochrane Database Syst Rev. 2009:CD006647.

2. Lipshultz SE, Adams MJ, Colan SD, Constine LS, Herman EH, Hsu DT, et al. Long-term cardiovascular toxicity in children, adolescents, and young adults who receive cancer therapy: pathophysiology, course, monitoring, management, prevention, and research directions: a scientific statement from the American Heart Association. Circulation. 2013;128:1927-95.

3. Feijen EAM, Leisenring WM, Stratton $\mathrm{KL}$, Ness KK, van der Pal HJH, van Dalen EC, et al. Derivation of anthracycline and anthraquinone equivalence ratios to doxorubicin for late-onset cardiotoxicity. JAMA Oncol. 2019;5:864-71.

4. Rasanen M, Degerman J, Nissinen TA, Miinalainen I, Kerkela R, Siltanen A et al. VEGF-B gene therapy inhibits doxorubicin-induced cardiotoxicity by endothelial protection. Proc Natl Acad Sci USA. 2016;113:13144-9.

5. Yu AF, Steingart RM, Fuster V. Cardiomyopathy associated with cancer therapy. J Card Fail. 2014;20:841-52.

6. Hortobagyi GN. Anthracyclines in the treatment of cancer. An overview. Drugs. 1997:54(Suppl 4):1-7.
7. Singal PK, Iliskovic N. Doxorubicin-induced cardiomyopathy. N Engl J Med. 1998:339:900-5

8. Young RC, Ozols RF, Myers CE. The anthracycline antineoplastic drugs. N Engl J Med. 1981;305:139-53.

9. Nitiss KC, Nitiss JL. Twisting and ironing: doxorubicin cardiotoxicity by mitochondrial DNA damage. Clin Cancer Res. 2014;20:4737-9.

10. Bry M, Kivela R, Leppanen VM, Alitalo K. Vascular endothelial growth factor-B in physiology and disease. Physiol Rev. 2014;94:779-94.

11. Heusch G, Libby P, Gersh B, Yellon D, Bohm M, Lopaschuk G, et al. Cardiovascular remodelling in coronary artery disease and heart failure. Lancet. 2014;383:1933-43.

12. Ichikawa Y, Ghanefar M, Bayeva M, Wu R, Khechaduri A, Naga Prasad SV, et al. Cardiotoxicity of doxorubicin is mediated through mitochondrial iron accumulation. J Clin Invest. 2014;124:617-30.

13. Wu CK, Lee JK, Hsu JC, Su MM, Wu YF, Lin TT, et al. Myocardial adipose deposition and the development of heart failure with preserved ejection fraction. Eur J Heart Fail. 2020;22:445-54.

14. Kumar D, Kirshenbaum LA, Li T, Danelisen I, Singal PK. Apoptosis in adriamycin cardiomyopathy and its modulation by probucol. Antioxid Redox Signal. 2001;3:135-45.

15. Li K, Sung RY, Huang WZ, Yang M, Pong NH, Lee SM, et al. Thrombopoietin protects against in vitro and in vivo cardiotoxicity induced by doxorubicin. Circulation. 2006;113:2211-20.

16. Pacher P, Liaudet L, Bai P, Virag L, Mabley JG, Hasko G, et al. Activation of poly(ADP-ribose) polymerase contributes to development of doxorubicin-induced heart failure. J Pharmacol Exp Ther. 2002;300:862-7.

17. Ueno M, Kakinuma Y, Yuhki K, Murakoshi N, lemitsu M, Miyauchi T, et al. Doxorubicin induces apoptosis by activation of caspase-3 in cultured cardiomyocytes in vitro and rat cardiac ventricles in vivo. J Pharmacol Sci. 2006;101:151-8.

18. Wu S, Ko YS, Teng MS, Ko YL, Hsu LA, Hsueh C, et al. Adriamycin-induced cardiomyocyte and endothelial cell apoptosis: in vitro and in vivo studies. J Mol Cell Cardiol. 2002;34:1595-607.

19. Laflamme MA, Murry CE. Heart regeneration. Nature. 2011;473:326-35.

20. Segers VF, Lee RT. Stem-cell therapy for cardiac disease. Nature. 2008:451:937-42

21. Roberts RM, Fisher SJ. Trophoblast stem cells. Biol Reprod. 2011:84:412-21.

22. Li G, Chen J, Zhang X, He G, Tan W, Wu H, et al. Cardiac repair in a mouse model of acute myocardial infarction with trophoblast stem cells. Sci Rep. 2017;7:44376.

23. Jung JH, Fu X, Yang PC. Exosomes generated from iPSC-derivatives: new direction for stem cell therapy in human heart diseases. Circ Res. 2017;120:407-17.

24. Kishore R, Khan M. More than tiny sacks: stem cell exosomes as cell-free modality for cardiac repair. Circ Res. 2016;118:330-43.

25. Aryal B, Jeong J, Rao VA. Doxorubicin-induced carbonylation and degradation of cardiac myosin binding protein $C$ promote cardiotoxicity. Proc Natl Acad Sci USA. 2014;111:2011-6.

26. Schickel R, Park SM, Murmann AE, Peter ME. miR-200c regulates induction of apoptosis through CD95 by targeting FAP-1. Mol Cell. 2010;38:908-15.

27. Xu L, Chen W, Ma M, Chen A, Tang C, Zhang C, et al. Microarray profiling analysis identifies the mechanism of miR-200b-3p/mRNA-CD36 affecting diabetic cardiomyopathy via peroxisome proliferator activated receptorgamma signaling pathway. J Cell Biochem. 2019;120:5193-206.

28. Lorenzen J, Kumarswamy R, Dangwal S, Thum T. MicroRNAs in diabetes and diabetes-associated complications. RNA Biol. 2012;9:820-7.

29. Williams $L$, Nye BG, Wende AR. Diabetes-related cardiac dysfunction. Endocrinol Metab (Seoul). 2017;32:171-9.

30. Gioffre S, Ricci V, Vavassori C, Ruggeri C, Chiesa M, Alfieri l, et al. Plasmatic and chamber-specific modulation of cardiac microRNAs in an acute model of DOX-induced cardiotoxicity. Biomed Pharmacother. 2019;110:1-8.

31. Pakravan G, Foroughmand AM, Peymani M, Ghaedi K, Hashemi MS, Hajjar $M$, et al. Downregulation of miR-130a, antagonized doxorubicin-induced cardiotoxicity via increasing the PPARgamma expression in $\mathrm{mESCs}$ derived cardiac cells. Cell Death Dis. 2018;9:758. 
32. Bei Y, Wu X, Cretoiu D, Shi J, Zhou Q, Lin S, et al. miR-21 suppression prevents cardiac alterations induced by d-galactose and doxorubicin. J Mol Cell Cardiol. 2018;115:130-41.

33. Ruggeri C, Gioffre S, Achilli F, Colombo Gl, D'Alessandra Y. Role of microRNAs in doxorubicin-induced cardiotoxicity: an overview of preclinical models and cancer patients. Heart Fail Rev. 2018;23:109-22.

34. Holmgren G, Synnergren J, Andersson CX, Lindahl A, Sartipy P. MicroRNAs as potential biomarkers for doxorubicin-induced cardiotoxicity. Toxicol In Vitro. 2016:34:26-34.

35. Magenta A, Cencioni C, Fasanaro P, Zaccagnini G, Greco S, Sarra-Ferraris G, et al. miR-200c is upregulated by oxidative stress and induces endothelial cell apoptosis and senescence via ZEB1 inhibition. Cell Death Differ. 2011;18:1628-39.

36. Korpal M, Lee ES, Hu G, Kang Y. The miR-200 family inhibits epithelialmesenchymal transition and cancer cell migration by direct targeting of E-cadherin transcriptional repressors ZEB1 and ZEB2. J Biol Chem. 2008:283:14910-4.

37. Luo SS, Ishibashi O, Ishikawa G, Ishikawa T, Katayama A, Mishima T, et al. Human villous trophoblasts express and secrete placenta-specific microRNAs into maternal circulation via exosomes. Biol Reprod. 2009;81:717-29.
38. Atay S, Gercel-Taylor C, Suttles J, Mor G, Taylor DD. Trophoblast-derived exosomes mediate monocyte recruitment and differentiation. Am J Reprod Immunol. 2011;65:65-77.

39. Alam SMK, Jasti S, Kshirsagar SK, Tannetta DS, Dragovic RA, Redman CW, et al. Trophoblast glycoprotein (TPGB/5T4) in human placenta: expression, regulation, and presence in extracellular microvesicles and exosomes. Reprod Sci. 2018;25:185-97.

40. Hou G, Dick R, Abrams GD, Brewer GJ. Tetrathiomolybdate protects against cardiac damage by doxorubicin in mice. J Lab Clin Med. 2005;146:299-303.

41. Renu K, Abilash VG, TirupathiPichiah PB, Arunachalam S. Molecular mechanism of doxorubicin-induced cardiomyopathy — an update. Eur J Pharmacol. 2018:818:241-53.

\section{Publisher's Note}

Springer Nature remains neutral with regard to jurisdictional claims in published maps and institutional affiliations.
Ready to submit your research? Choose BMC and benefit from:

- fast, convenient online submission

- thorough peer review by experienced researchers in your field

- rapid publication on acceptance

- support for research data, including large and complex data types

- gold Open Access which fosters wider collaboration and increased citations

- maximum visibility for your research: over $100 \mathrm{M}$ website views per year

At BMC, research is always in progress.

Learn more biomedcentral.com/submissions 